\title{
Metodologia e perfil sociodemográfico, cognitivo e de fragilidade de idosos comunitários de sete cidades brasileiras: Estudo FIBRA
}

\begin{abstract}
Methodology and social, demographic, cognitive, and frailty profiles of community-dwelling elderly from seven Brazilian cities: the FIBRA Study
\end{abstract}

Metodología y perfil sociodemográfico, cognitivo y de fragilidad de ancianos en comunidades desfavorecidas de siete ciudades brasileñas: Estudio FIBRA

\author{
Anita Liberalesso Neri 1 \\ Mônica Sanches Yassuda ${ }^{2}$ \\ Ludgleydson Fernandes de Araújo 3 \\ Maria do Carmo Eulálio 4 \\ Benedita Edina Cabral 5 \\ Maria Eliane Catunda de Siqueira 6 \\ Geraldine Alves dos Santos 7 \\ José Guilherme de Arruda Moura ${ }^{8}$
}

\section{Resumo}

${ }^{1}$ Faculdade de Ciências Médicas, Universidade Estadual de Campinas, Campinas, Brasil.

2 Escola de Artes, Ciências e Humanidades, Universidade de São Paulo, São Paulo, Brasil.

${ }^{3}$ Departamento de Psicologia, Universidade Federal do Piauí, Parnaíba, Brasil.

${ }^{4}$ Centro de Ciências Biológicas e da Saúde, Universidade da Paraíba, Campina Grande, Brasil. ${ }_{5}$ Centro de Humanidades, Universidade Federal de Campina Grande, Campina Grande, Brasil. ${ }_{6}$ Pontifícia Universidade Católica de Minas Gerais, Poços de Caldas, Brasil. 7 Curso de Psicologia Universidade Feevale Novo Hamburgo, Brasil. 8 Secretaria de Estado de Saúde Pública do Estado do Pará, Belém, Brasil.

Correspondência A. L. Neri

Programa de Pós-graduação em Gerontologia, Faculdade de Ciências Médicas, Universidade Estadual de Campinas.

Av. Tessalia Vieira de Camargo

126, Campinas, $S P$

13083-887, Brasil.

anitalbn@uol.com.br

\section{Abstract}

A study was designed to identify conditions of frailty in relation to social, demographic, health, cognitive, functional, and psychosocial variables in community-dwelling elderly. The article presents the methodology and preliminary data. A total of 3,478 elderly (65 years and older) were selected from probabilistic samples of seven Brazilian cities chosen by convenience and participated in a data collection session in a community setting. The following characteristics predominated: women (67.7\%), married (48\%) or widowed (36.4\%), living with a son or daughter and family (52.6\%), head of family (64.5\%), and 1-4 years of schooling (49\%); $28.8 \%$ were illiterate and $24.8 \%$ presented a cognitive deficit; $9.1 \%$ were frail, 51.8\% pre-frail, and 39.1\% non-frail. There were more frail individuals among women, those 80 years or older, the widowed, the illiterate, those who had never attended school, and those with cognitive deficit. In general, the social and demographic data corroborate Brazilian epidemiological studies, while those on frailty, cognitive status, and schooling corroborate the international literature.

Frail Elderly; Health of the Elderly; Health Vulnerability; Health Surveys
Um estudo foi planejado para identificar condições de fragilidade em relação a variáveis sociodemográficas, de saúde, cognição, funcionalidade e psicossociais em idosos comunitários. Metodologia e dados preliminares são apresentados. Foram selecionados 3.478 idosos (65 anos e mais), integrantes de amostras probabilísticas de sete cidades brasileiras escolhidas por conveniência, participaram de sessão de coleta de dados, em ambiente comunitário. Predominaram as seguintes características: mulheres (67,7\%), casados (48\%) ou viúvos (36,4\%), vivendo com a família de filho/a (52,6\%), chefes de família (64,5\%) $e$ 1-4 anos de escolaridade (49\%); 28,8\% eram analfabetos e 24,8\% tinham déficit cognitivo; 9,1\% eram frágeis, 51,8\% pré-frágeis e 39,1\% não-frágeis. Houve mais frágeis entre as mulheres, os de 80 anos e mais, os viúvos, os analfabetos, os que nunca foram à escola e os com déficit cognitivo. Em geral, os dados sociodemográficos replicam os de estudos epidemiológicos brasileiros, e os de fragilidade, estado cognitivo e escolaridade, os da literatura internacional.

Idoso Fragilizado; Saúde do Idoso; Vulnerabilidade em Saúde; Inquéritos Epidemiológicos 


\section{Introdução}

O ponto de vista mais difundido sobre fragilidade é o que a define como síndrome clínica geriátrica envolvendo declínio das reservas de energia, desregulação neuroendócrina, rebaixamento da função imune e diminuição da resistência a estressores, resultantes do processo normativo de envelhecimento fisiológico, ou senescência, em interação com riscos atuais e acumulados à saúde e a funcionalidade. Indivíduos frágeis compõem um subconjunto de idosos portadores de maior susceptibilidade a desfechos adversos de saúde, tais como morte, incapacidade e hospitalização em virtude da redução de sua capacidade para responder a condições de estresse, vulnerabilidade que também os predispõe a doenças crônicas, anorexia, sarcopenia, a osteopenia, déficits cognitivos e incapacidade 1,2 .

A definição operacional de fragilidade considerada como síndrome geriátrica compreende cinco critérios derivados de dois estudos prospectivos, o Cardiovascular Health Study (CHS) 1 e o Women's Health and Aging Study (WHAS) 2: (a) autorrelato de perda de peso não intencional no último ano, igual ou superior a $4,5 \mathrm{~kg}$ ou a 5\% do peso corporal; (b) fadiga indicada pelas respostas sempre ou quase sempre a dois itens da Center for Epidemiological Study - Depression (CES-D) ${ }^{3}$ que perguntam sobre queda percebida no nível de energia na última semana; (c) baixa taxa de gasto calórico semanal em exercícios físicos e em atividades domésticas autorrelatados - pontuam para este critério aqueles cuja taxa de gasto calórico em kcal localiza-se abaixo do 1o quintil da amostra (com ajuste por sexo); (d) baixa força de preensão, quando a média em $\mathrm{kg}_{\mathrm{f}}$ de três medidas consecutivas tomadas com dinamômetro hidráulico manual, modelo Jamar (Sahean, Masan, Coréia do Sul), localiza-se abaixo do lo quintil da amostra (com ajustes por IMC e sexo); (e) lentidão da marcha, quando a média de três medidas consecutivas do tempo (em segundos) para percorrer $4,6 \mathrm{~m}$ no plano e em passo usual, é superior ao percentil $80 \mathrm{da}$ amostra (as médias são ajustadas por sexo e altura). Pontuação em três ou mais critérios significa fragilidade, em um ou dois significa pré-fragilidade e, em nenhum, não-fragilidade.

No CHS, que envolveu idosos residentes na comunidade, $6,3 \%$ dos participantes foram classificados como frágeis, $45,3 \%$ como pré-frágeis e $48,3 \%$ como não-frágeis. A incidência de fragilidade em quatro anos foi de $7,2 \%$. Fragilidade associou-se com raça negra, baixos níveis de escolaridade e renda, saúde deficitária e altas taxas de comorbidades e incapacidade. A prevalência foi maior entre as mulheres do que entre os ho- mens e entre os idosos de idade avançada do que entre os idosos jovens (32\% no grupo de 90 anos ou mais versus $2,5 \%$ entre os de 65 a 70 anos). Dos idosos classificados como frágeis $59 \%$ foram hospitalizados em três anos, contra 33\% dos não frágeis; $28 \%$ dos frágeis e $15 \%$ dos não frágeis sofreram quedas; $39 \%$ dos frágeis e $8 \%$ dos não frágeis apresentaram piora nas atividades básicas de vida diária e $18 \%$ dos frágeis morreram, contra $3 \%$ dos não frágeis. Os pré-frágeis apresentaram risco intermediário para esses desfechos e maior risco de se tornarem frágeis em 3 a 4 anos, quando comparados com os não-frágeis e aos frágeis $1,2,4$.

Condições de risco tais como ser mulher, não ser de raça branca, baixa renda, baixo nível educacional, acesso deficitário a serviços de saúde e viver sozinho são apontadas como preditores de fragilidade 5,6,7,8. Avila-Funes et al. ${ }^{9}$ observaram prevalência e incidência significativamente maiores de déficit cognitivo entre os idosos frágeis do que entre os pré-frágeis e os não-frágeis de três cidades francesas. Os frágeis que tinham déficit cognitivo apresentaram maior tendência para incapacidade em atividades instrumentais de vida diária (AIVD) e em atividades básicas de vida diária (ABVD) nos quatro anos subsequentes. A incidência de demência foi maior entre aqueles com déficit cognitivo, independentemente do nível de fragilidade. Várias pesquisas apontaram o déficit cognitivo como variável relacionada à incidência de fragilidade 10,11,12 e de doença de Alzheimer 13,14.

A diminuição da resiliência biológica e da plasticidade comportamental compatíveis com a senescência e com a fragilidade acarretam aumento de perdas em saúde, funcionalidade física, funcionamento intelectual, atividade, motivação, participação social e bem-estar subjetivo 15 , as quais podem ser agravadas por eventos estressantes gerados pela vulnerabilidade social 16, entre eles as necessidades não atendidas 17, e pelo duplo prejuízo de ser idoso e pertencer ao segmento social dos mais pobres e menos educados. A escassez de oportunidades sociais ao longo da vida aumenta os riscos de fragilidade 5,6,7,8. No entanto, existe documentação sobre resiliência física expressa em menor incidência de fragilidade em grupos de idosos pré-frágeis, com diabetes, comorbidades, baixa renda e baixa escolaridade 18,19,20,21 .

Não há dados brasileiros com idosos com 65 anos e mais correlacionando fragilidade, condições de saúde física e mental, condições psicossociais e variáveis demográficas e socioeconômicas. Os objetivos deste trabalho foram: (a) descrever a metodologia do Estudo FIBRA Unicamp, uma investigação de caráter descriti- 
vo, comparativo e de corte transversal envolvendo amostras probabilísticas de idosos residentes em sete localidades brasileiras; (b) caracterizar a amostra conforme variáveis demográficas e socioeconômicas e conforme indicadores de fragilidade e de estado cognitivo; (c) identificar similaridades e diferenças quanto às frequências de idosos frágeis, pré-frágeis e não-frágeis, conforme variáveis sociodemográficas selecionadas e estado cognitivo.

\section{Métodos}

FIBRA é o acrônimo de Fragilidade em Idosos Brasileiros e designa uma rede de pesquisa dedicada a identificar condições de fragilidade em idosos urbanos recrutados na comunidade, com 65 anos e mais, e a investigar relações dos indicadores de fragilidade com variáveis demográficas e socioeconômicas e com aspectos selecionados da saúde física e da cognição, desempenho de atividades de vida diária e expectativa de cuidado, com sintomas depressivos e satisfação com a vida.

Essa rede organizou-se em quatro pólos, cada um dos quais nucleou os trabalhos de um grupo de instituições parceiras. Em cada pólo, certo número de cidades, bairros ou subdistritos foram selecionados por conveniência, atendendo a um dos requisitos do edital do Conselho Nacional de Desenvolvimento Científico e Tecnológico (CNPq), que determinava que um grupo experiente em pesquisa sobre o envelhecimento deveria atrair outros grupos com menor experiência, de preferência sediados nas regiões Norte e Nordeste (Edital MCT-CNPq/MS - SCTIE-DECIT, no 17/2006). Integraram o pólo Unicamp (Universidade Estadual de Campinas) as localidades de Campinas (São Paulo), Belém (Pará), Parnaíba (Piauí), Campina Grande (Paraíba), Poços de Caldas (Minas Gerais) e Ivoti (Rio Grande do Sul) e o subdistrito de Ermelino Matarazzo em São Paulo. As instituições parceiras da Unicamp foram a Secretaria de Estado de Saúde Pública do Pará (SESPA); a Universidade Federal do Piauí (UFPI); a Universidade Federal de Campina Grande (UFCG) e a Universidade Estadual da Paraíba (UEPB); a Pontifícia Universidade Católica de Minas Gerais em Poços de Caldas (PUC-MG); a Escola de Artes, Ciências e Humanidades da Universidade de São Paulo (EACH/USP) e a Fundação Feevale em Novo Hamburgo (Rio Grande do Sul).

As condições de vida das populações dessas sete localidades espelham os contrastes socioeconômicos existentes entre as regiões Norte e Nordeste, menos desenvolvidos, e as regiões
Sudeste e Sul, as mais ricas do país, e também dentro da Região Sudeste (Tabela 1).

\section{Amostragem e participantes}

Em cada uma das localidades foi feita amostragem aleatória simples de setores censitários urbanos (Instituto Brasileiro de Geografia e Estatística. http://www.ibge.gov.br), cujo número correspondeu à razão entre o número de idosos pretendidos e o número de setores censitários urbanos. O número de setores censitários sorteados e percorridos pelos recrutadores foi o seguinte: 90 em Campinas, 93 em Belém, 75 em Poços de Caldas, 62 em Ermelino Matarazzo, 60 em Campina Grande e 60 em Parnaíba e 27 (todos) em Ivoti. Em cada amostra foram estimadas cotas de homens e mulheres de 65 a 69, 70 a 74, 75 a 79 e 80 anos e mais, respeitando-se a proporcionalidade da distribuição desses segmentos na população idosa residente na zona urbana.

O tamanho mínimo da amostra estimada para Campinas e Belém, que têm mais de 1 milhão de habitantes, foi de 601 idosos, para um erro amostral de $4 \%$. Para as demais, com menos de 1 milhão de habitantes, a estimativa foi de 384 idosos, para um erro amostral de 5\%. Em Ivoti, para um universo de 646 idosos houve uma estimativa de 235 indivíduos, com base na consideração de uma população finita, alfa fixado em $5 \%$ e erro amostral de $5 \%$.

\section{Recrutamento}

$\mathrm{O}$ recrutamento compreendeu duas fases. $\mathrm{Na}$ primeira, que antecedeu as visitas domiciliares, foram feitos anúncios nas emissoras de rádio e TV e nos jornais. Líderes religiosos e comunitários, coordenadores de centros de convivência e agentes de saúde foram envolvidos na divulgação, depois de serem informados quanto aos objetivos e aos cuidados éticos da pesquisa. Os coordenadores locais e a coordenadora geral da pesquisa realizaram palestras de sensibilização dirigidas ao público idoso em geral e aos docentes e alunos das universidades envolvidas.

A segunda fase do recrutamento compreendeu visitas aos domicílios por duplas de recrutadores, que foram previamente treinados conforme as regras estabelecidas no manual de recrutamento construído para a pesquisa. Todos eram identificados por um crachá contendo nome e fotografia, além de bolsa, camiseta, pasta, formulários, canetas, cartões de visita e folhetos de divulgação da pesquisa com elementos de identificação visual da Rede FIBRA, da Unicamp e da instituição parceira. Os recrutadores anunciavam sua presença em todos os domicílios das 
Tabela 1

Cotas de idosos estimadas e obtidas, por idade e gênero, nas sete localidades identificadas pelos respectivos Índice de Desenvolvimento Humano Municipal (IDH-M) e o produto interno bruto (PIB) per capita. Estudo FIBRA Unicamp, Brasil, 2008-2009.

\begin{tabular}{|c|c|c|c|c|c|c|c|c|c|c|c|c|}
\hline \multirow{2}{*}{$\begin{array}{l}\text { Cidade/Estado * } \\
\text { [habitantes] ** }\end{array}$} & \multirow[t]{2}{*}{ IDH-M ** } & \multirow{2}{*}{$\begin{array}{c}\text { PIB per } \\
\text { capita } \\
(\mathrm{R} \$)^{\star \star \star}\end{array}$} & \multirow{2}{*}{$\begin{array}{l}\text { Idades } \\
\text { (anos) }\end{array}$} & \multicolumn{3}{|c|}{ Homens (1.125) } & \multicolumn{3}{|c|}{ Mulheres (2.353) } & \multicolumn{3}{|c|}{ Total (3.478) } \\
\hline & & & & $\begin{array}{c}\text { Estimadas } \\
(\%)\end{array}$ & $\begin{array}{c}\text { Obtidas } \\
\text { (\%) }\end{array}$ & $\begin{array}{l}\text { Valor } \\
\text { de } p\end{array}$ & $\begin{array}{c}\text { Estimadas } \\
\text { (\%) }\end{array}$ & $\begin{array}{l}\text { Obtidas } \\
\text { (\%) }\end{array}$ & $\begin{array}{l}\text { Valor } \\
\text { de } p\end{array}$ & $\begin{array}{c}\text { Estimadas } \\
\text { (\%) }\end{array}$ & $\begin{array}{l}\text { Obtidas } \\
\text { (\%) }\end{array}$ & $\begin{array}{l}\text { Valor } \\
\text { de } p\end{array}$ \\
\hline Belém/PA & 0,797 & $9.793,00$ & $65-69$ & 38,5 & 33,2 & $0,366 \#$ & 34,4 & 38,1 & 0,000 & 35,9 & 36,6 & 0,009 \\
\hline \multirow[t]{3}{*}{ [1.393.399] } & & & $70-74$ & 28,3 & 30,5 & & 26,9 & 27,5 & & 27,4 & 28,4 & \\
\hline & & & $75-79$ & 17,5 & 17,7 & & 17,0 & 21,2 & & 17,3 & 20,1 & \\
\hline & & & $80+$ & 15,7 & 18,6 & & 21,7 & 13,2 & & 19,4 & 14,8 & \\
\hline Parnaíba/PI & 0,674 & $5.484,85$ & $65-69$ & 30,3 & 28,9 & $0,896 \#$ & 31,2 & 37,3 & 0,006 & 30,8 & 34,3 & $0,105 \#$ \\
\hline \multirow[t]{3}{*}{ [145.705] } & & & $70-74$ & 26,4 & 28,3 & & 26.6 & 24,4 & & 26,5 & 25,8 & \\
\hline & & & $75-79$ & 21,7 & 20,2 & & 19,0 & 22,2 & & 20,1 & 21,5 & \\
\hline & & & $80+$ & 21,6 & 22,5 & & 23.2 & 16,1 & & 22,6 & 18,4 & \\
\hline Campina Grande/PB & 0,721 & $10.147,21$ & $65-69$ & 30,3 & 29,8 & $0,366 \#$ & 34,1 & 30,1 & 0,001 & 34,5 & 30,0 & 0,004 \\
\hline \multirow[t]{3}{*}[385.213]{} & & & $70-74$ & 26,4 & 28,9 & & 27,3 & 32,6 & & 27,4 & 31,5 & \\
\hline & & & $75-79$ & 21,7 & 14,9 & & 17,8 & 19,1 & & 18,1 & 17,9 & \\
\hline & & & $80+$ & 21,6 & 26,4 & & 20,8 & 18,1 & & 20,0 & 20,6 & \\
\hline Ermelino Matarazzo/SP & 0,730 & & $65-69$ & 41,5 & 38,1 & $0,896 \#$ & 37,5 & 38,0 & $0,347 \#$ & 39,2 & 38,0 & $0,678 \#$ \\
\hline \multirow[t]{3}{*}[207.509]{} & & & $70-74$ & 29,6 & 30,2 & & 28,3 & 32,2 & & 28,8 & 31,5 & \\
\hline & & & $75-79$ & 16,7 & 17,5 & & 17,7 & 16,7 & & 17,2 & 17,0 & \\
\hline & & & $80+$ & 12,2 & 14,3 & & 16,5 & 13,2 & & 14,8 & 13,5 & \\
\hline Poços de Caldas/MG & 0,841 & $19.137,52$ & $65-69$ & 38,7 & 28,0 & 0,051 \# & 35,0 & 39,3 & 0,067 \# & 36,5 & 35,0 & $0,051 \#$ \\
\hline \multirow[t]{3}{*}[152.435]{} & & & $70-74$ & 28,3 & 35,3 & & 27,8 & 28,9 & & 28,0 & 31,4 & \\
\hline & & & $75-79$ & 17,2 & 20,0 & & 18,0 & 129 & & 17,7 & 19,5 & \\
\hline & & & $80+$ & 15,8 & 16,7 & & 19,2 & 12,6 & & 17,8 & 14,1 & \\
\hline Campinas/SP & 0,852 & $29.731,00$ & $65-69$ & 39,1 & 31,5 & 0,010 & 34,2 & 35,6 & 0,050 & 36,3 & 34,3 & 0,001 \\
\hline \multirow[t]{3}{*}{ [1.083.113] } & & & $70-74$ & 28,7 & 38,4 & & 28,0 & 28,8 & & 28,2 & 31,8 & \\
\hline & & & $75-79$ & 17,0 & 18,5 & & 18,4 & 21,5 & & 17,9 & 20,6 & \\
\hline & & & $80+$ & 15,2 & 11,6 & & 19,4 & 14,1 & & 17,6 & 13,3 & \\
\hline Ivoti/RS & 0,851 & $17.967,69$ & $65-69$ & 46,1 & 32,2 & $0,091 \#$ & 34,1 & 33,3 & 0,001 & 38,7 & 33,0 & 0,002 \\
\hline \multirow[t]{3}{*}{ [19.874] } & & & $70-74$ & 23,5 & 35,6 & & 22,8 & 35,5 & & 23,1 & 35,5 & \\
\hline & & & $75-79$ & 20,7 & 20,3 & & 19,3 & 20,3 & & 19,8 & 20,3 & \\
\hline & & & $80+$ & 9,7 & 11,9 & & 23,8 & 10,9 & & 18,4 & 11,2 & \\
\hline
\end{tabular}

MG: Minas Gerais; PA: Pará; PB: Paraíba; PI: Piauí; RS: Rio Grande do Sul; SP: São Paulo.

* O tamanho mínimo estimado para a amostra das localidades com mais de 1 milhão de habitantes (Belém e Campinas) foi de 601 idosos. Para as que tinham menos que 1 milhão, a amostra mínima foi estimada em 384 idosos. A amostra de lvoti, que tinha cerca de 600 idosos, foi estimada em 235 . As amostras obtidas foram: Belém = 721; Parnaíba = 484; Campina Grande = 403; Poços de Caldas = 389; Ermelino Matarazzo = 384; Campinas = 900 e lvoti = 197; ** Dados do Instituto Brasileiro de Geografia e Estatística. Síntese de indicadores sociais: uma análise das condições de vida da população brasileira. http://www.ibge.gov.br (acessado em 30/Abr/2012);

*** Dados do Programa das Nações Unidas para o Desenvolvimento. Ranking do IDHM dos municípios do Brasil, ano 2000. http://www.pnud.org.br/atlas/ tabelas (acessado em 30/Abr/2012);

\# Se $p \geq 0,05$, as cotas esperadas e obtidas são estatisticamente equivalentes (teste chi-quadrado), se $p<0,05$, as cotas esperadas e obtidas são estatisticamente diferentes (teste de qui-quadrado).

ruas percorridas, numa sequência previamente demarcada num mapa e investigavam se neles havia um ou mais idosos que cumpriam os critérios de inclusão (ter idade igual ou superior a 65 anos, compreender as instruções, concordar em participar da pesquisa e ser residente per- manente no domicilio e no setor censitário). Os recrutadores foram orientados a utilizar os seguintes critérios de exclusão: presença de problemas de memória, atenção, orientação espacial, temporal e comunicação sugestivos de déficit cognitivo; incapacidade permanente ou 
temporária para andar, exceto com uso de dispositivo de auxílio à marcha; perda localizada de força e afasia decorrentes de sequela de acidente vascular encefálico (AVE); comprometimento grave da motricidade, da fala ou da afetividade associados à doença de Parkinson avançada; déficit auditivo ou visual grave e estar em estágio terminal. Os informantes podiam ser os próprios idosos ou um familiar residente no domicílio. Os critérios de inclusão e exclusão foram baseados em Fried et al. 1 e Ferrucci et al. 2. Nome, gênero, idade, endereço e telefone dos idosos recrutados, bem como dados de identificação de um familiar para contato eram anotados em fichas de registro. Fichas com endereço, local, data e horário da coleta de dados, telefone e nome para contato foram oferecidas aos idosos por ocasião do recrutamento.

O recrutamento e a coleta de dados foram feitos em ondas sucessivas. Em cada uma, os idosos eram recrutados em número que satisfizesse as estimativas do plano amostral para um ou mais setores censitários adjacentes e eram encaminhados para a coleta de dados. Cada onda era encerrada quando os idosos esperados haviam sido submetidos às medidas.

\section{Coleta de dados}

Os dados foram coletados em sessão única, em escolas, igrejas, unidades básicas de saúde, centros de conveniência e clubes, por estudantes de graduação e de pós-graduação. Todos receberam treinamento oferecido por equipe oriunda da Unicamp. Havia um manual de coleta de dados a ser seguido, o qual foi oferecido a cada um dos colaboradores. As sessões duravam de 40 a 120 minutos. No início, os idosos eram informados sobre os objetivos da pesquisa, o caráter voluntário de sua participação, o direito a abandonar a pesquisa a qualquer momento, o sigilo dos dados individuais e a ausência de riscos à saúde física e mental e eram convidados a assinar um Termo de Consentimento Livre e Esclarecido (TCLE) Junto com o projeto, este foi aprovado pelo Comitê de Ética em Pesquisa com Seres Humanos da Faculdade de Ciências Médicas da Unicamp, sob o parecer no $208 / 2007$.

Os idosos eram recebidos pelo coordenador e encaminhados para um dos entrevistadores, para a realização de um dos cinco blocos da primeira fase da coleta de dados, que incluiu medidas de variáveis demográficas e socioeconômicas, antropométricas, pressão arterial, fragilidade e rastreio cognitivo. Em Campinas foram também investigados os medicamentos para tratamento de diabetes e hipertensão e foi realizado exame clínico da cavidade oral.
A cada bloco terminado, os idosos deslocavam-se até outro entrevistador e assim sucessivamente até o final dos cinco blocos iniciais de medida. O entrevistador que completava o último bloco da primeira fase de coleta de dados, verificava a pontuação do idoso no Mini Exame do Estado Mental (MEEM) 22. Caso o escore obtido fosse superior à nota de corte, o idoso progredia para a segunda parte do protocolo, que incluiu medidas de autorrelato de condições de saúde física, funcionalidade, expectativa de cuidado, sintomas depressivos e satisfação com a vida. Caso o resultado fosse inferior à nota de corte, o idoso era dispensado antes de realizar o segundo bloco de medidas. Ao final de sua sessão de coleta de dados cada idoso recebia orientações sobre cuidados à saúde e uma cartilha de saúde.

As notas de corte utilizadas para exclusão pelo MEEM foram: 17 para os analfabetos; 22 para idosos com escolaridade entre 1 e 4 anos; 24 para os com escolaridade entre 5 e 8 anos e 26 para os que tinham 9 anos ou mais anos de escolaridade. Estes pontos de corte foram baseados nos critérios sugeridos pela Academia Brasileira de Neurologia, com base em Brucki et al. 23, menos um desvio padrão. A opção por tais notas de corte, também adotada por Fried et al. 1, Ferrucci et al. 2 e Bandeen-Roche et al. 4 foi feita porque déficits cognitivos poderiam prejudicar a confiabilidade das respostas de autorrelato.

Na segunda parte da coleta de dados, as variáveis investigadas por meio de medidas de autorrelato foram: doenças crônicas, sinais e sintomas, problemas de sono, quedas e fraturas, uso de medicamentos, dificuldades visuais e auditivas, tabagismo e alcoolismo e avaliação subjetiva de saúde; acesso a serviços médicos e hospitalares no último ano; percepção sobre a saúde bucal e sobre as condições funcionais para a alimentação; funcionalidade indicada pelo desempenho de atividades avançadas de vida diária (AAVD), AIVDs e ABVDs; expectativa de cuidado; sintomas depressivos e satisfação.

Além dessas, foram avaliadas as seguintes variáveis psicossociais: autoeficácia para quedas (Belém e Poços de Caldas); experiência de eventos estressantes (Campinas, Parnaíba e Ivoti); estratégias de enfrentamento e percepção de eficácia do enfrentamento (Parnaíba e Ivoti); afetos positivos e negativos e senso de ajustamento psicológico (Campina Grande); suporte social percebido (Campinas, Ermelino Matarazzo, Ivoti, Parnaíba e Campina Grande); nomeação, memória incidental, memória imediata e aprendizado; fluência verbal - categoria animais; desenho do relógio e autoavaliação de memória; percepção de velhice e autoimagem (Ermelino Matarazzo); significados atribuídos à 
"velhice saudável" (Belém, Parnaíba, Poços de Caldas, Ermelino Matarazzo e Ivoti), e significados atribuídos ao conceito "ser feliz na velhice" (Campinas).

\section{Análise de dados}

Antes da digitação dos dados em bancos eletrônicos, os protocolos eram sucessivamente conferidos por dois supervisores. Foi feita conferência dos dados digitados por dois avaliadores treinados, com exigência de $100 \%$ de acordo.

As análises estatísticas foram feitas por meio do SPSS, versão 15.1 (SPSS Inc., Chicago, Estados Unidos), e do SAS, versão 8.02 (SAS Inst., Cary, Estados Unidos). Estatísticas descritivas foram realizadas para explorar os dados. O estudo das relações entre as variáveis foi feito por meio de testes de correlação bivariada e de modelos adequados de regressão, entre eles o linear, o logístico e o hierárquico. As análises de conglomerados foram realizadas pelo método de partição. Os instrumentos de natureza escalar tiveram seus itens submetidos à análise de consistência interna, assumindo-se como indicador o alfa de Cronbach.

Para o presente relato foram selecionadas análises de frequência, posição e dispersão e comparações entre frequências de variáveis entre grupos mediante testes chi-quadrado. A margem de erro aceita para todos os testes estatísticos foi de $5 \%$.

\section{Resultados}

A amostra das sete localidades totalizou 3.478 idosos (67,7\% mulheres); $34,6 \%$ dos idosos tinham de 65 a 69 anos; $30,5 \%$ de 70 a $74 ; 19,7 \%$ de 75 a 79 e 15,2\%, 80 anos e mais. Com base em testes chi-quadrado, observou-se equivalência estatística entre as amostras esperadas e obtidas de homens pertencentes aos quatro grupos de idade em todas as localidades, com exceção de Campinas. Em Belém, Parnaíba, Campina Grande, Campinas e Ivoti as cotas de mulheres conforme as idades foram superiores ao esperado. Houve equivalência entre as cotas estimadas e obtidas de mulheres em Ermelino Matarazzo e em Poços de Caldas. Na amostra total, as localidades de Parnaíba, Ermelino Matarazzo e Poços de Caldas tiveram amostras de homens e mulheres dos quatro grupos de idade estatisticamente equivalentes ao esperado. Na Tabela 1 são apresentados os valores das cotas de idosos estimadas e obtidas conforme gênero e idade. As localidades são identificadas conforme a população, o Índice de Desenvolvimento Humano
Municipal (IDH-M) e o produto interno bruto (PIB) per capita (Tabela 1).

As mulheres foram estatisticamente mais numerosas em Ivoti e em Campina Grande; os homens em Poços de Caldas; $65,1 \%$ da amostra tinham entre 65 e 74 anos, mas não foi observada diferença estatisticamente significativa entre as médias de idade das sete localidades. Metade dos idosos da amostra total se autodescreveram como brancos. Porém, as localidades com nível mais elevado de desenvolvimento econômico tiveram um percentual de brancos significativamente mais alto do que aquelas com nível de desenvolvimento mais baixo: $97,5 \%$ dos idosos de Ivoti, 73,6\% dos de Poços de Caldas e 70,9\% de Campinas, as cidades com PIB per capita mais alto da amostra declararam-se brancos. Em contrapartida as frequências para essas variáveis em Belém e Parnaíba foram $24,4 \%$ e $30,3 \%$, respectivamente. Os percentuais mais altos de pardos foram observados em Belém (63,3\%), Parnaíba (59,4\%) e Campina Grande (51,7\%).

O maior número de casados foi encontrado em Ivoti, Poços de Caldas e Campinas. Os percentuais de idosos viúvos foram mais altos em Ivoti, Parnaíba e Campina Grande. Os maiores percentuais de idosos que declararam morar sozinhos foram encontrados em Ivoti e Poços de Caldas e os que viviam apenas com o cônjuge, também nessas duas cidades, denotando melhor situação financeira e, possivelmente, melhor saúde. Belém, Campina Grande e Parnaíba apresentaram as maiores frequências de idosos que viviam com a família de filho e em arranjos mistos, denotando arranjos multigeracionais e ampliados. Os percentuais mais baixos desses tipos de arranjos domiciliares foram observados em Ivoti, Poços de Caldas e Campinas (Tabela 2).

Foram observadas frequências significativamente mais altas de idosos que se declararam analfabetos em Parnaíba e Campina Grande. Em Ivoti foi observado o maior percentual de alfabetizados. Parnaíba e Campina Grande tiveram os percentuais mais elevados de idosos sem escolaridade formal, em contraste com Ivoti. Em Ermelino Matarazzo, Poços de Caldas, Ivoti e Campinas foram encontrados os maiores percentuais de idosos com 1 a 4 anos de escolaridade, em contraste com Parnaíba, Campina Grande e Belém, cidades com nível socioeconômico mais baixo. Ivoti teve a maior frequência de idosos com 5 a 8 anos de escolaridade; Belém e Parnaíba os maiores percentuais dos que fizeram curso colegial e Poços de Caldas e Campinas, a maior frequência de idosos que frequentaram curso superior.

Campina Grande, Belém e Campina Grande apresentaram os contingentes mais numerosos de idosos com renda individual menor ou igual 
Idosos das sete localidades conforme indicadores demográficos. Estudo FIBRA Unicamp, Brasil, 2008-2009.

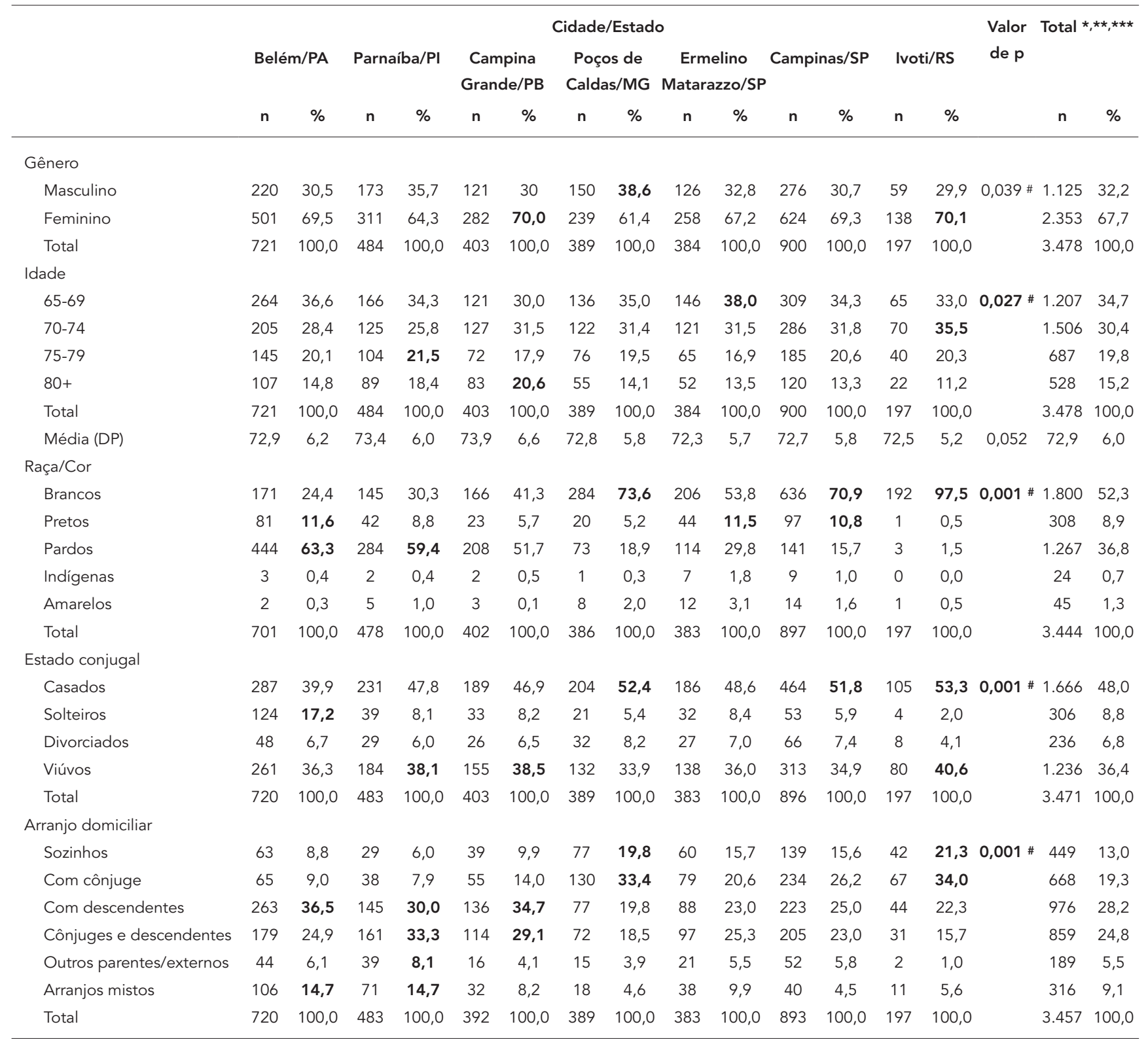

DP: desvio-padrão; MG: Minas Gerais; PA: Pará; PB: Paraíba; PI: Piauí; RS: Rio Grande do Sul; SP: São Paulo.

* O tamanho mínimo estimado para a amostra das localidades com mais de um milhão de habitantes (Belém e Campinas) foi de 601 idosos. Para as que tinham menos que um milhão, a amostra mínima foi estimada em 384 idosos. A amostra de Ivoti, que tinha cerca de 600 idosos, foi estimada em 235 . As amostras obtidas foram: Belém = 721; Parnaíba = 484; Campina Grande = 403; Poços de Caldas = 389; Ermelino Matarazzo $=384 ;$ Campinas $=900$ e Ivoti $=197 ;$

** Dados do Instituto Brasileiro de Geografia e Estatística. Síntese de indicadores sociais: uma análise das condições de vida da população brasileira. http://www.ibge.gov.br (acessado em 30/Abr/2012);

*** As diferenças nas frequências em raça, estado conjugal e arranjo domiciliar, em comparação com gênero e idade, são relativas ao total de casos de ausência de respostas em cada uma delas;

\# Se $p \leq 0,05$ (teste de qui-quadrado). 
a um salário mínimo; Ivoti e Ermelino Matarazzo abrigavam as maiores frequências relativas de idosos com valores de renda individual entre 1,1 e 3,0 salários mínimos e Campinas a maior frequência de idosos com renda pessoal entre 1,1 e 3,0 salários mínimos. Em Campinas e Poços de Caldas foi observado o maior percentual de idosos que declararam ter renda individual superior a 5,0 salários mínimos. As rendas familiares mais altas foram observadas em Campinas. Os percentuais mais elevados de idosos com renda familiar $\leq 1$ estavam novamente em Campina Grande, Parnaíba e Belém e os percentuais mais altos de idosos com renda familiar de 1,1 a 3,0 salários mínimos em Parnaíba, Ermelino Matarazzo, Campina Grande e Belém.

Em Ivoti residia o maior percentual de idosos que declararam que a renda era suficiente para enfrentar os gastos do domicílio; em Belém e Campina Grande, o maior percentual dos que opinaram que a renda era insuficiente. Os percentuais mais altos dos que declararam que eram os principais responsáveis pelo sustento da família residiam em Parnaíba (78,5\%) e Campina Grande (71,4\%). A grande maioria dos idosos de todas as localidades eram proprietários da residência e eram aposentados. Uma minoria estatisticamente significante declarou que trabalhava (Tabela 3 ).

Na Tabela 4 são mostrados os dados de frequência para os indicadores de fragilidade e de estado cognitivo. Foram encontradas diferenças estatisticamente significantes entre os percentuais de idosos que pontuaram para perda de peso não-intencional (o menor valor em Ivoti e o maior em Parnaíba) e para fadiga (os maiores valores em Parnaíba e Campina Grande e os menores em Campinas e Belém). No entanto, os percentuais de idosos que pontuaram para baixa força de preensão, lentidão da marcha e inatividade física foram estatisticamente comparáveis nas sete localidades, assim como os percentuais de frágeis, pré-frágeis e não-frágeis. Na amostra total, cerca de metade dos idosos pontuou para a condição de pré-fragilidade e a outra metade dividiu-se entre a condição de não-fragilidade e a de fragilidade. Oitocentos e oitenta e três idosos $(25,4 \%$ do total) pontuaram abaixo da nota de corte no MEEM. Os maiores percentuais de idosos que pontuaram para déficit cognitivo foram observados em Campina Grande e Parnaíba, as localidades com PIB per capita mais baixo da amostra. Os menores foram observados em Poços de Caldas e em Ivoti, que se colocavam entre as três com os melhores níveis de renda.

Houve significativamente mais mulheres do que homens que pontuaram para fragilidade e mais homens não-frágeis do que mulheres. Fo- ram observados significantemente mais idosos de 80 anos do que das outras faixas de idade e maior percentual de idosos pré-frágeis entre os de 75 anos e mais. Entre os de 65 a 69, foi observado o percentual de idosos significantemente menor entre os quatro grupos de idade. Houve significantemente mais idosos frágeis entre os analfabetos e os sem escolaridade formal do que entre os alfabetizados e os que frequentaram escola. Percentuais comparáveis de idosos pré-frágeis foram observados entre os sem escolaridade formal e entre os com 1 a 4 anos de escolaridade. Entre os não-frágeis foram observados os percentuais significantemente mais altos de idosos com 5 a 11 anos de escolaridade. Houve frequência relativa significantemente mais alta de idosos que não eram chefes de domicílio e que julgavam que a renda da família não era suficiente para cobrir os gastos do que entre os que declararam que eram chefes ou que os recursos eram suficientes (Tabela 5).

\section{Discussão}

O Estudo FIBRA é a primeira investigação de natureza multicêntrica sobre fragilidade conduzida com idosos brasileiros de 65 anos e mais. Embora as localidades tenham sido selecionadas por critério de conveniência, o fato de as amostras serem probabilísticas terá prevenido a ocorrência de vieses sistemáticos de seleção. O fato de espelharem condições socioeconômicas contrastantes terá propiciado mais variabilidade e sobreposição ao real. No entanto, as discrepâncias observadas entre as cotas estimadas e as obtidas por localidade, conforme os critérios de sexo e idade, representam uma limitação à possibilidade de generalizar amplamente os resultados para as populações, pelo menos antes de serem calculados os graus de influência dessas distorções sobre os resultados. Da mesma forma, a maior frequência de mulheres do que sua presença na população terá sido uma limitação à generalidade dos dados.

A participação de mais mulheres do que homens do que o planejado foi provavelmente fruto de o recrutamento ter sido feito principalmente por mulheres, a maioria das quais eram agentes comunitárias de saúde, que provavelmente desfrutavam de mais acesso e confiança dos participantes potenciais do que se fossem homens e desconhecidos. As mulheres costumam ter presença mais assídua em amostras de pesquisas envolvendo saúde, em virtude de sua condição de cuidadoras e da sua maior exposição a tratamentos de saúde ao longo da vida 23. É provável que as recrutadoras tenham comparecido aos 
Idosos das sete localidades conforme indicadores socioeconômicos. Estudo FIBRA Unicamp, Brasil, 2008-2009.

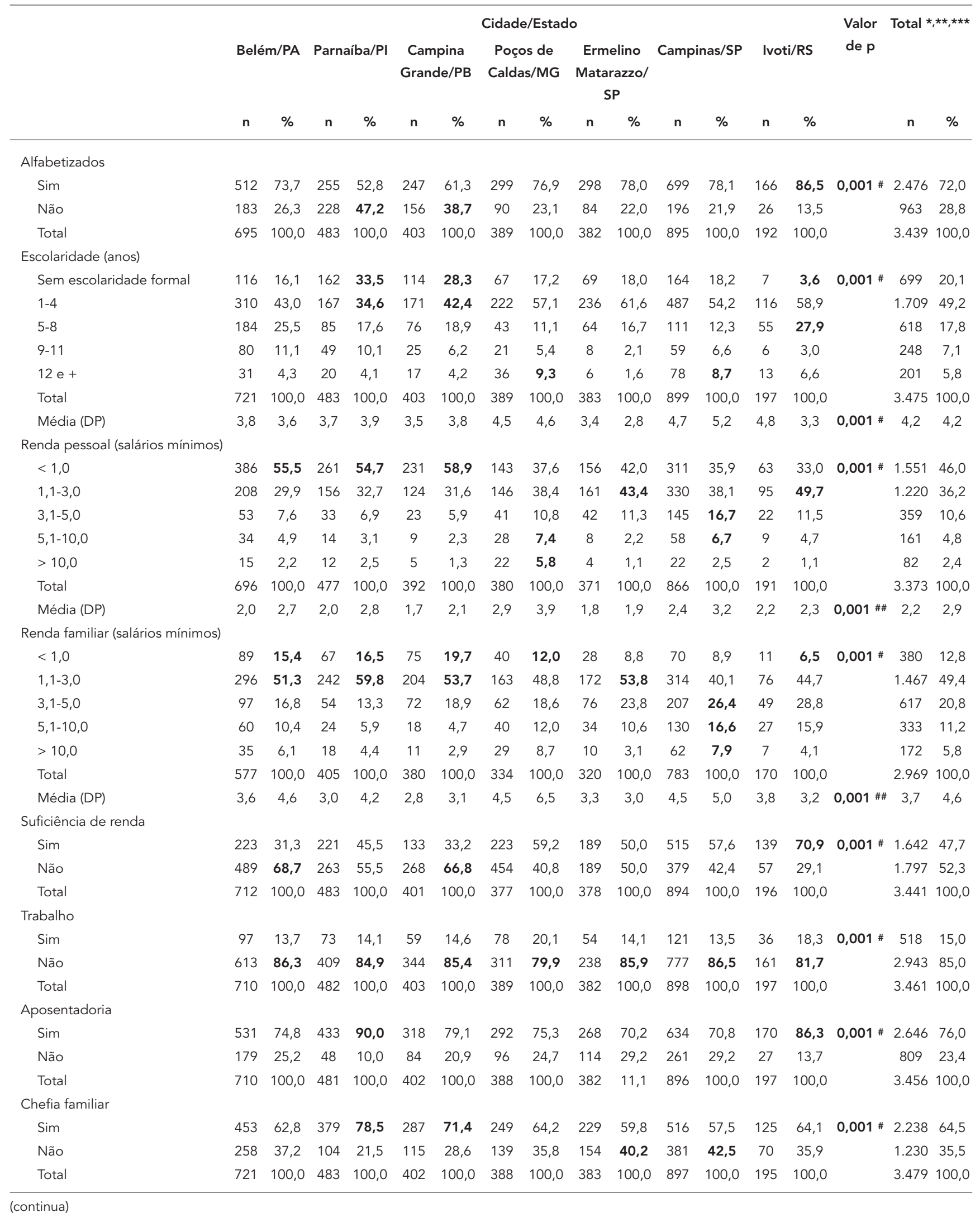




\begin{tabular}{|c|c|c|c|c|c|c|c|c|c|c|c|c|c|c|c|c|c|}
\hline & \multicolumn{14}{|c|}{ Cidade/Estado } & \multirow{3}{*}{$\begin{array}{l}\text { Valor } \\
\text { de } p\end{array}$} & \multicolumn{2}{|c|}{ Total $\star, \star \star, \star \star *$} \\
\hline & \multicolumn{2}{|c|}{ Belém/PA } & \multicolumn{2}{|c|}{ Parnaíba/PI } & \multicolumn{2}{|c|}{$\begin{array}{l}\text { Campina } \\
\text { Grande/PB }\end{array}$} & \multicolumn{2}{|c|}{$\begin{array}{c}\text { Poços de } \\
\text { Caldas/MG }\end{array}$} & \multicolumn{2}{|c|}{$\begin{array}{c}\text { Ermelino } \\
\text { Matarazzo/ } \\
\text { SP }\end{array}$} & \multicolumn{2}{|c|}{ Campinas/SP } & \multicolumn{2}{|c|}{ Ivoti/RS } & & & \\
\hline & $\mathbf{n}$ & $\%$ & $\mathbf{n}$ & $\%$ & $\mathrm{n}$ & $\%$ & $\mathrm{n}$ & $\%$ & $\mathrm{n}$ & $\%$ & $\mathbf{n}$ & $\%$ & $\mathbf{n}$ & $\%$ & & $\mathrm{n}$ & $\%$ \\
\hline \multicolumn{18}{|c|}{ Propriedade do domicílio } \\
\hline Sim & 604 & 83,8 & 395 & 81,6 & 309 & 76,8 & 267 & 68,6 & 276 & 72,3 & 738 & 82,0 & 164 & 83,2 & $0,001 \#$ & 2.753 & 79,2 \\
\hline Não & 117 & 16,2 & 89 & 18,4 & 94 & 23,2 & 122 & 31,4 & 107 & 27,7 & 162 & 18,0 & 33 & 16,8 & & 724 & 20,8 \\
\hline Total & 721 & 100,0 & 484 & 100,0 & 403 & 100,0 & 389 & 100,0 & 383 & 100,0 & 900 & 100,0 & 197 & 100,0 & & 3.477 & 100,0 \\
\hline
\end{tabular}

MG: Minas Gerais; PA: Pará; PB: Paraíba; PI: Piauí; RS: Rio Grande do Sul; SP: São Paulo.

* O tamanho mínimo estimado para a amostra das localidades com mais de um milhão de habitantes (Belém e Campinas) foi de 601 idosos. Para as que tinham menos que um milhão, a amostra mínima foi estimada em 384 idosos. A amostra de Ivoti, que tinha cerca de 600 idosos, foi estimada em 235 As amostras obtidas foram: Belém = 721; Parnaíba = 484; Campina Grande = 403; Poços de Caldas = 389; Ermelino Matarazzo = 384; Campinas = 900 e Ivoti $=197$;

** Dados do Instituto Brasileiro de Geografia e Estatística. Síntese de indicadores sociais: uma análise das condições de vida da população brasileira. http://www.ibge.gov.br (acessado em 30/Abr/2012);

*** As somas dos casos apresentam diferenças entre as variáveis devido à existência de um número variável de não-respostas; \# Se $p \leq 0,05$; teste de qui-quadrado;

\#\# Se $p \leq 0,05$; teste de Kruskal-Wallis.

domicílios em horário comercial, quando os homens têm maior probabilidade de estarem fora de casa, dedicando-se a atividades de trabalho ou lazer externas à vida doméstica, o que explicaria a adesão de menor número de homens do que o esperado. As cotas de idosos muito idosos mais reduzidas do que as previamente estabelecidas deveram-se possivelmente à exigência de deslocamento dos participantes até o local de coleta de dados, que terá sido de cumprimento mais difícil pelos idosos mais velhos.

Foram tomados cuidados para evitar distorções sistemáticas aos dados, por exemplo, por meio do encorajamento à participação dos idosos; da padronização dos procedimentos, instrumentos e equipamentos e pelo exaustivo treinamento das equipes de recrutamento e coleta de dados em todas as localidades, e pelos procedimentos adotados para assegurar maior confiabilidade aos dados inseridos nos bancos eletrônicos. No entanto, a perda de informações por ocasião da coleta de dados foi uma limitação à confiabilidade dos dados, assim como a perda de participantes, principalmente em Ivoti onde a amostra foi menor que o esperado, por recusas em comparecer à sessão de coleta de dados por alegados problemas de horário e de transporte. Futuras pesquisas deverão investir na maior clareza dos itens do protocolo com potencial para gerar dúvidas ou resistências (como por exemplo, renda), em formas de contornar desistências e no planejamento de cotas de participantes para reposição de perdas.
As frequências por critérios de cor ou raça, renda individual e familiar, chefia familiar, propriedade da residência, trabalho e aposentadoria nas sete localidades foram comparáveis aos dados censitários do ano de 200922 e aos de estudos epidemiológicos brasileiros 19,23. Da mesma forma, as frequências de idosos frágeis, pré-frágeis e não-frágeis foram similares aos encontrados na literatura internacional 1,4,5,7,8,13,14, assim como as relações entre fragilidade e gênero feminino, idade avançada, baixa renda, analfabetismo e baixa escolaridade 5,6,7,8,13,14. A associação entre fragilidade e déficit cognitivo; de cada uma dessas variáveis com analfabetismo e com baixa escolaridade, e de fragilidade com a percepção de que as próprias necessidades não são atendidas pelos rendimentos, igualmente replicam o observado em estudos internacionais 7,15,16. Refletem desvantagens acumuladas ao longo do curso de vida, às quais se somam as perdas associadas ao envelhecimento.

A seleção de idosos sem déficit cognitivo e a exigência de comparecimento dos idosos por seus próprios meios ao local de coleta de dados terão inserido um viés de sobrevivência no estudo, ou seja, a possibilidade de participação majoritária de idosos bem-sucedidos em termos físicos, emocionais e cognitivos. São desconhecidos os efeitos dessa decisão sobre os resultados, uma vez que a amostra não foi comparada com outras compostas por idosos entrevistados em casa, hospitais ou instituições de longa permanência. A exclusão dos idosos com déficit cognitivo 
Idosos das sete localidades conforme indicadores de fragilidade e de déficit cognitivo. Estudo FIBRA Unicamp, Brasil, $2008-2009$.

\begin{tabular}{|c|c|c|c|c|c|c|c|c|c|c|c|c|c|c|c|c|c|}
\hline & \multicolumn{14}{|c|}{ Cidade/Estado } & \multirow{3}{*}{$\begin{array}{l}\text { Valor } \\
\text { de p }\end{array}$} & \multicolumn{2}{|c|}{ 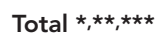 } \\
\hline & \multicolumn{2}{|c|}{ Belém/PA } & \multicolumn{2}{|c|}{ Parnaíba/PI } & \multicolumn{2}{|c|}{$\begin{array}{l}\text { Campina } \\
\text { Grande/PB }\end{array}$} & \multicolumn{2}{|c|}{$\begin{array}{c}\text { Poços de } \\
\text { Caldas/MG }\end{array}$} & \multicolumn{2}{|c|}{$\begin{array}{c}\text { Ermelino } \\
\text { Matarazzo/SP }\end{array}$} & \multicolumn{2}{|c|}{ Campinas/SP } & \multicolumn{2}{|c|}{ Ivoti/RS } & & & \\
\hline & $\mathrm{n}$ & $\%$ & $\mathrm{n}$ & $\%$ & $\mathrm{n}$ & $\%$ & $\mathrm{n}$ & $\%$ & $\mathrm{n}$ & $\%$ & $\mathrm{n}$ & $\%$ & $\mathrm{n}$ & $\%$ & & $\mathrm{n}$ & $\%$ \\
\hline \multicolumn{18}{|l|}{ Perda de peso } \\
\hline Sim & 141 & 20,7 & 90 & 25,5 & 71 & 19,2 & 82 & 21,6 & 74 & 19,6 & 145 & 16,5 & 17 & 8,7 & $0,001 \#$ & 620 & 19,1 \\
\hline Não & 544 & 79,4 & 273 & 74,5 & 299 & 80,8 & 297 & 78,4 & 304 & 80,4 & 732 & 83,5 & 178 & 91,3 & & 2.627 & 80,9 \\
\hline Total & 685 & 100,0 & 363 & 100,0 & 370 & 100,0 & 379 & 100,0 & 378 & 100,0 & 877 & 100,0 & 195 & 100,0 & & 3.247 & 100,0 \\
\hline \multicolumn{18}{|l|}{ Fadiga } \\
\hline Sim & 132 & 19,2 & 120 & 28,7 & 104 & 28,7 & 89 & 23,3 & 89 & 23,4 & 172 & 19,3 & 41 & 23,8 & $0,001 \#$ & 752 & 22,7 \\
\hline Não & 555 & 80,8 & 298 & 71,3 & 258 & 71,3 & 293 & 76,7 & 292 & 76,6 & 720 & 80,7 & 147 & 76,2 & & 2.563 & 77,3 \\
\hline Total & 687 & 100,0 & 418 & 100,0 & 362 & 100,0 & 382 & 100,0 & 381 & 100,0 & 892 & 100,0 & 193 & 100,0 & & 3.315 & 100,0 \\
\hline \multicolumn{18}{|c|}{ Baixa força de preensão } \\
\hline Sim & 151 & 21,2 & 88 & 20,5 & 76 & 19,1 & 83 & 21,4 & 78 & 20,6 & 180 & 20,1 & 40 & 20,6 & 0,987 & 696 & 20,5 \\
\hline Não & 562 & 78,8 & 342 & 79,5 & 322 & 80,9 & 305 & 78,6 & 301 & 79,4 & 714 & 79,9 & 154 & 79,4 & & 2.700 & 79,5 \\
\hline Total & 713 & 100,0 & 430 & 100,0 & 398 & 100,0 & 388 & 100,0 & 379 & 100,0 & 894 & 100,0 & 194 & 100,0 & & 3.396 & 100,0 \\
\hline \multicolumn{18}{|c|}{ Lentidão da marcha } \\
\hline Sim & 144 & 20,0 & 87 & 20,1 & 72 & 19,7 & 77 & 19,8 & 76 & 19,8 & 176 & 19,9 & 40 & 20,3 & 1,000 & 672 & 19,9 \\
\hline Não & 577 & 80,0 & 346 & 79,9 & 293 & 80,3 & 311 & 80,2 & 308 & 80,2 & 707 & 80,1 & 157 & 79,7 & & 2.699 & 80,1 \\
\hline Total & 721 & 100,0 & 433 & 100,0 & 365 & 100,0 & 388 & 100,0 & 384 & 100,0 & 783 & 100,0 & 164 & 100,0 & & 3.371 & 100,0 \\
\hline \multicolumn{18}{|c|}{ Inatividade física } \\
\hline Sim & 143 & 20,1 & 86 & 20,0 & 79 & 19,8 & 79 & 20,4 & 79 & 20,7 & 179 & 20,0 & 40 & 20,3 & 1,000 & 685 & 20,1 \\
\hline Não & 568 & 79,9 & 344 & 80,0 & 319 & 80,2 & 308 & 79,6 & 302 & 79,3 & 718 & 80,0 & 157 & 79,7 & & 2.716 & 79,9 \\
\hline Total & 721 & 100,0 & 430 & 100,0 & 365 & 100,0 & 387 & 100,0 & 381 & 100,0 & 897 & 100,0 & 197 & 100,0 & & 3.401 & 100,0 \\
\hline \multicolumn{18}{|c|}{ Níveis de fragilidade } \\
\hline Frágeis & 78 & 10,8 & 42 & 9,7 & 35 & 8,9 & 36 & 9,3 & 31 & 8,1 & 69 & 7,7 & 17 & 8,6 & 0,280 & 308 & 9,0 \\
\hline Pré-frágeis & 347 & 48,2 & 239 & 55,5 & 203 & 51,4 & 207 & 53,4 & 211 & 54,9 & 469 & 52,2 & 94 & 47,7 & & 1.770 & 51,9 \\
\hline Não-frágeis & 295 & 41,0 & 150 & 34,8 & 157 & 39,7 & 145 & 37,4 & 142 & 37,0 & 360 & 40,1 & 86 & 43,7 & & 1.335 & 39,1 \\
\hline Total & 720 & 100,0 & 431 & 100,0 & 395 & 100,0 & 388 & 100,0 & 384 & 100,0 & 898 & 100,0 & 197 & 100,0 & & 3.413 & 100,0 \\
\hline \multicolumn{18}{|l|}{ Déficit cognitivo } \\
\hline Sim & 145 & 20,1 & 185 & 38,2 & 153 & 38,1 & 71 & 18,3 & 81 & 21,1 & 211 & 23,9 & 37 & 18,8 & & 883 & 25,4 \\
\hline Não & 576 & 79,9 & 299 & 61,8 & 249 & 61,9 & 318 & 81,7 & 302 & 78,9 & 689 & 76,6 & 160 & 81,2 & $0,001 \#$ & 2.593 & 74,6 \\
\hline Total & 721 & 100,0 & 484 & 100,0 & 402 & 100,0 & 389 & 100,0 & 383 & 100,0 & 900 & 100,0 & 197 & 100,0 & & 3.476 & 100,0 \\
\hline
\end{tabular}

MG: Minas Gerais; PA: Pará; PB: Paraíba; PI: Piauí; RS: Rio Grande do Sul; SP: São Paulo.

* O tamanho mínimo estimado para a amostra das localidades com mais de um milhão de habitantes (Belém e Campinas) foi de 601 idosos. Para as que tinham menos que um milhão, a amostra mínima foi estimada em 384 idosos. A amostra de Ivoti, que tinha cerca de 600 idosos, foi estimada em 235. As amostras obtidas foram: Belém = 721; Parnaíba = 484; Campina Grande = 403; Poços de Caldas = 389; Ermelino Matarazzo = 384; Campinas = 900 e Ivoti $=197$;

** Dados do Instituto Brasileiro de Geografia e Estatística. Síntese de indicadores sociais: uma análise das condições de vida da população brasileira. http://www.ibge.gov.br (acessado em 30/Abr/2012);

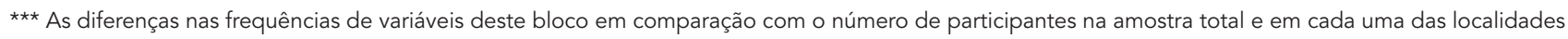
são devidas à ausência de respostas;

\# Se $\mathrm{p} \leq 0,05$; teste de qui-quadrado.

sugestivo de demência na segunda parte da coleta de dados replicou o procedimento adotado por Fried et al. 1 e Ferrucci et al. 2, cujo objetivo foi favorecer a fidedignidade dos dados de autorrelato. Neste estudo pretendeu-se também conhecer como os indicadores de fragilidade funcionavam em amostras de idosos brasileiros.

Embora sem a intenção de considerar as localidades como representativas dos diversos níveis de desenvolvimento econômico existentes 
Tabela 5

Idosos frágeis, pré-frágeis e não-frágeis conforme variáveis demográficas e socioeconômicas e estado cognitivo. Estudo FIBRA Unicamp, Brasil, 2008-2009.

\begin{tabular}{|c|c|c|c|c|c|c|c|c|c|}
\hline & \multicolumn{2}{|c|}{ Frágeis } & \multicolumn{2}{|c|}{ Pré-frágeis } & \multicolumn{2}{|c|}{ Não-frágeis } & \multirow{2}{*}{$\begin{array}{l}\text { Valor } \\
\text { de } p\end{array}$} & \multicolumn{2}{|c|}{ Total } \\
\hline & $\mathrm{n}$ & $\%$ & $\mathrm{n}$ & $\%$ & $\mathbf{n}$ & $\%$ & & $\mathrm{n}$ & $\%$ \\
\hline \multicolumn{10}{|l|}{ Gênero } \\
\hline Masculino & 84 & 7,6 & 564 & 51,0 & 458 & 41,4 & 0,046 * & 1.106 & 32,4 \\
\hline Feminino & 224 & 9,7 & 1.206 & 52,3 & 877 & 38,0 & & 2.307 & 67,6 \\
\hline Total & 308 & 9,0 & 1.770 & 51,9 & 1.335 & 39,1 & & 3.413 & 100,0 \\
\hline \multicolumn{10}{|l|}{ Idade (anos) } \\
\hline $65-69$ & 53 & 4,5 & 567 & 47,7 & 568 & 47,8 & 0,001 * & 1.188 & 34,8 \\
\hline $70-74$ & 74 & 7,1 & 535 & 51,6 & 428 & 41,3 & & 1.037 & 30,4 \\
\hline $75-79$ & 80 & 11,8 & 375 & 55,5 & 221 & 32,7 & & 676 & 19,8 \\
\hline $80+$ & 101 & 19,7 & 293 & 57,2 & 118 & 23,0 & & 512 & 15,0 \\
\hline Total & 308 & 9,0 & 1.770 & 51,9 & 1.335 & 39,1 & & 3.413 & 100,0 \\
\hline \multicolumn{10}{|l|}{ Estado conjugal } \\
\hline Casados & 124 & 7,6 & 828 & 50,5 & 686 & 41,9 & 0,001 * & 1.638 & 48,1 \\
\hline Solteiros & 29 & 9,6 & 157 & 51,8 & 117 & 38,6 & & 303 & 8,9 \\
\hline Divorciados & 12 & 5,2 & 108 & 47,2 & 109 & 47,6 & & 229 & 6,7 \\
\hline Viúvos & 142 & 11,5 & 673 & 54,4 & 421 & 34,1 & & 1.236 & 36,3 \\
\hline Total & 307 & 9,0 & 1666 & 51,9 & 1333 & 39,1 & & 3.406 & 100,0 \\
\hline \multicolumn{10}{|l|}{ Analfabetos } \\
\hline Sim & 140 & 15,2 & 1.238 & 55,4 & 271 & 29,2 & 0,001 * & 922 & 27,3 \\
\hline Não & 163 & 6,6 & 511 & 50,5 & 1.051 & 42,7 & & 2.452 & 72,7 \\
\hline Total & 303 & 9,0 & 1.749 & 51,9 & 1.322 & 39,1 & & 3.374 & 100,0 \\
\hline \multicolumn{10}{|l|}{ Escolaridade (anos) } \\
\hline Sem escolaridade formal & 104 & 15,5 & 362 & 53,9 & 205 & 30,6 & $0,001 *$ & 671 & 19,7 \\
\hline $1-4$ & 136 & 8,1 & 895 & 53,1 & 656 & 38,9 & & 1.687 & 49,5 \\
\hline $5-8$ & 48 & 7,9 & 292 & 47,9 & 270 & 44,3 & & 610 & 17,9 \\
\hline $9-11$ & 9 & 3,7 & 122 & 50,4 & 111 & 45,9 & & 242 & 7,1 \\
\hline $12+$ & 10 & 5,0 & 98 & 49,0 & 92 & 46,0 & & 200 & 5,9 \\
\hline Total & 307 & 9,0 & 1.769 & 51,9 & 1.334 & 39,1 & & 3.410 & 100,0 \\
\hline \multicolumn{10}{|l|}{ Chefia familiar } \\
\hline Sim & 178 & 8,1 & 1144 & 52,3 & 865 & 39,6 & 0,031 * & 2.187 & 64,2 \\
\hline Não & 127 & 10,4 & 622 & 51,2 & 467 & 38,4 & & 1.216 & 35,7 \\
\hline Total & 305 & 9,0 & 1766 & 51,9 & 1.332 & 39,1 & & 3.404 & 100,0 \\
\hline \multicolumn{10}{|l|}{ Dinheiro suficiente } \\
\hline Sim & 131 & 8,1 & 821 & 50,8 & 664 & 41,1 & 0,001 * & 1.616 & 48,0 \\
\hline Não & 175 & 10,0 & 926 & 52,7 & 656 & 37,3 & & 1.758 & 52,0 \\
\hline Total & 306 & 9,1 & 1.747 & 51,8 & 1.320 & 39,1 & & 3.376 & 100,0 \\
\hline \multicolumn{10}{|l|}{ Estado cognitivo } \\
\hline Com déficit & 126 & 15,3 & 461 & 56,0 & 236 & 28,7 & 0,001 * & 823 & 24,1 \\
\hline Sem déficit & 182 & 7,0 & 1.308 & 50,5 & 1.098 & 42,4 & & 2.588 & 75,9 \\
\hline Total & 308 & 9,0 & 1.769 & 51,9 & 1.334 & 39,1 & & 3.411 & 100,0 \\
\hline
\end{tabular}

* Se $p \leq 0,05$; teste de qui-quadrado. 
no país, foi interessante notar relações entre os indicadores de renda daquelas que detém PIB per capita mais baixos e PIB per capita mais altos. A presença de idosos que se descreveram como pardos e pretos, com baixa renda e baixa escolaridade, analfabetos, com déficit cognitivo, vivendo com os descendentes ou em arranjos mistos e com renda domiciliar julgada insuficiente para fazer frente às necessidades, foi significantemente maior nas primeiras do que nas últimas, sugerindo a vigência da acumulação de déficits em saúde, cognição e bem-estar ao longo da vida, em virtude da privação continuada de benefícios e oportunidades e da desigualdade social 7,16,17,18,24. Nas três últimas, foi maior a frequência de idosos brancos, com renda e escolaridade mais altas, de menos analfabetos, de menor número de idosos com déficit cognitivo e de maior número de idosos vivendo em arranjos conjugais ou sozinhos, replicando dados da literatura internacional que apontam renda e escolaridade como variáveis de proteção em relação à má qualidade de vida em saúde e a déficits cognitivos 17,18

Os indicadores de fragilidade associados ao envelhecimento fisiológico (baixa força de preensão e lentidão da marcha) não se apresentaram de forma diferente nas diferentes localidades, sugerindo que respondem a variáveis menos afetadas por estados subjetivos (caso da fadiga, que é também indicador de depressão) ou por nível educacional e de renda (caso da inatividade física). Quase $60 \%$ dos idosos foram identificados como frágeis ou pré-frágeis, de forma comparável ao observado em estudos internacionais que envolveram idosos recrutados na comunidade. Porem, a prevalência de perda de peso não-intencional no último ano foi muito maior neste estudo do que a registrada na literatura $1,2,4,13$. Especula-se se esse resultado terá sido influenciado pelo formato da pergunta ou pela alta desejabilidade social de ter um corpo magro, que terá levado os respondentes a superestimarem o valor de uma eventual perda de peso.

Em todas as localidades, percentuais comparáveis de participantes pontuaram para baixa força nos membros superiores e inferiores, para baixo gasto calórico semanal em atividades físicas e para fadiga, independentemente das condições socioeconômicas das localidades de residência, confirmando dados internacionais $8,13,18$. Tal dado sugere que a influência do declínio fisiológico associado ao envelhecimento normal sobre a perda de força e de função muscular 1,2,4 prevaleceu sobre a influência de variáveis socioeconômicas.

\section{Conclusões}

Estudos epidemiológicos realizados em outros países têm mostrado que, somados, os idosos frágeis e pré-frágeis correspondem a cerca de metade das populações de 60 anos e mais 25 , como se viu neste estudo. A frequência de idosos que pontuaram para cada um dos cinco indicadores de fragilidade foi comparável à encontrada em estudos internacionais, sugerindo que a senescência tem papel importante na fragilização das populações idosas. Assim, os esforços de diagnóstico e intervenção precoces nos produtos do declínio normal e patológico do envelhecimento são, inquestionavelmente, uma importante questão de saúde pública.

Pensar que, para a grande maioria de idosos deste estudo, as relações entre pré-fragilidade, fragilidade e variáveis sociodemográficas ocorrem em contextos de pobreza e de baixa escolaridade, chama a atenção para os enormes desafios impostos às atuais e futuras coortes de idosos brasileiros, em busca de equilíbrio entre as condições oferecidas pela sociedade e aquelas que eles mesmos, suas famílias e suas redes sociais mais próximas podem engendrar em favor de um envelhecimento saudável.

$\mathrm{O}$ aparente paradoxo representado pela presença de um contingente expressivo de idosos pré-frágeis e frágeis que continuam ativos e participativos, mesmo em meio a privações e adversidades, como se viu neste e em outros estudos brasileiros 18,19,20,21, é um incentivo à investigação da influência de variáveis psicológicas sobre o alcance de boa qualidade de vida na velhice, mesmo na presença das perdas normativas do envelhecimento, em interação com os prejuízos econômicos, educacionais e de saúde acumulados ao longo do curso de vida. 


\section{Resumen}

Se planificó un estudio para identificar condiciones de fragilidad en relación con las variables sociodemográficas, de salud, cognición, funcionalidad y psicosociales en ancianos de comunidades desfavorecidas. En el estudio se presenta la metodología y datos preliminares. Fueron seleccionados 3.478 ancianos (65 años y más), integrantes de muestras probabilísticas de siete ciudades brasileñas, escogidas por conveniencia. En la sesión de recogida de datos participaron dentro de un ambiente de comunidades desfavorecidas. Predominaron las siguientes características: mujeres (67,7\%), casados (48\%) o viudos (36,4\%), viviendo con la familia de hijola (52,6\%), jefes de familia (64,5\%) y 1-4 años de escolaridad (49\%); un 28,8\% eran analfabetos y un 24,8\% tenían déficit cognitivo; un 9,1\% eran enfermizos; un $51,8 \%$ pre-enfermizos y un $39,1 \%$ no enfermizos. Hubo más enfermizos entre las mujeres, quienes tenían 80 años y más, los viudos, los analfabetos, los que nunca fueron a la escuela y quienes tienen déficit cognitivo. En general, los datos sociodemográficos replican los de estudios epidemiológicos brasileños, y los de morbilidad, status cognitivo y escolaridad, además de los de la literatura internacional.

Anciano Frágil; Salud del Anciano; Vulnerabilidad en Salud; Encuestas Epidemiológicas

\section{Referências}

1. Fried LP, Tangen CM, Walston J, Newman AB, Hirsch C, Gottdiener J, et al. Frailty in older adults: evidence for a phenotype. J Gerontol A Biol Sci Med Sci 2001; 56:M146-56.

2. Ferrucci L, Guralnik JM, Studenski S, Fried LP, Cutler Jr. GB, Walston JD. Designing randomized, controlled trials aimed at preventing or delaying functional decline and disability in frail, older persons: a consensus report. J Am Geriatr Soc 2004; 52:625-34.

3. Radloff L. The CES-D Scale: a self-report depression scale for research in the general population. Appl Psychol Meas 1977; 1:385-401.

4. Bandeen-Roche K, Xue QL, Ferrucci L, Walston J, Guralnik JM, Chaves P, et al. Phenotype of frailty: characterization in the women's health and aging studies. J Gerontol A Biol Sci Med Sci 2006; 61:262-6.

\section{Colaboradores}

A. L. Neri foi responsável pela análise e pela interpretação dos dados, bem como pela redação e pela aprovação final deste artigo para publicação. M. S. Yassuda colaborou na interpretação dos dados e na redação deste artigo, bem como reviu criticamente sua versão final. L. F. Araújo, M. C. Eulálio, B. E. Cabral, M. E. C. Siqueira, G. A. Santos e J. G. A. Moura opinaram sobre a análise de dados, colaboraram na redação do artigo e em sua revisão.

\section{Agradecimentos}

A Andréa Cristina Garofe Fortes-Burgos, Efigênia Passarelli Mantovani, Flavia Silva Arbex Borim, Stella Vital de Souza Torres, Débora Dias da Silva Harmitt, Natalia Oliveira Rodrigues, Juliana Martins Pinto, Cristina Rodrigues Lima, Helymar Machado; aos professores e estudantes de graduação e pós-graduação que coletaram dados nas sete cidades e aos bolsistas de graduação que digitaram os dados das sete cidades do pólo da Universidade Estadual de Campinas (Unicamp). Ao CNPq pelo financiamento.
5. Strawbridge WJ, Shema SJ, Balfour JL, Higby HR, Kaplan GA. Antecedents of frailty over three decades in an older cohort. J Gerontol B Psychol Sci 1998; 53:9-16.

6. Yang Y, Lee LC. Dynamics and heterogeneity in the process of human frailty and aging: evidence from the U. S. older adult population. J Gerontol B Psychol Sci 2010; 65:246-55.

7. Bilotta C, Bowling A, Nicolini P, Casè A, Vergani C. Quality of life in older outpatients living alone in the community in Italy. Health Soc Care Community 2012; 20:32-41.

8. Szanton SL, Seplaki CL, Thorpe Jr. RJ, Allen JK, Fried LP. Socioeconomic status is associated with frailty: the Women's Health and Aging Studies. J Epidemiol Community Health 2010; 64:63-7. 
9. Avila-Funes JA, Helmer C, Amieva H, BarbergerGateau D, Le Goff M, Ritche K, et al. Frailty among community-dwelling elderly people in France: the three-city study. J Gerontol A Biol Sci Med Sci 2009; 63:1089-96.

10. Sternberg SA, Wershof Schwartz A, Karunanthan S, Bergman H, Mark Clarfield A. The identification of frailty: a systematic literature review. J Am Ger Soc 2011; 59:2129-38.

11. Macuco CRM, Batistoni SST, Lopes A, Cachioni M, Falcão DVS, Neri AL, et al. Mini-Mental State Examination performance in frail, pre-frail and nonfrail community dwelling older adults in Ermelino Matarazzo, São Paulo, Brazil. Int Psychogeriatr 2012; 24:1725-31.

12. Yassuda MS, Lopes A, Cachioni M, Falcão DV, Batistoni, SS, Guimarães VV, et al. Frailty criteria and cognitive performance are related: data from the FIBRA Study in Ermelino Matarazzo, São Paulo, Brazil. J Nutr Health Aging 2012; 16:55-61.

13. Andrew MK, Rockwood K. Social vulnerability predicts cognitive decline in a prospective cohort of older Canadians. Alzheimers Dement 2010; 6 : 319-24.

14. Panza F, Solfrizzi V, Frisardi V, Maggi C, Sancarlo D, Adante F, et al. Different models of frailty in predementia and dementia syndromes. J Nutr Health Aging 2011; 15:711-9.

15. Agrigoroaei S, Lachman ME. Cognitive functioning in midlife and old age: combined effects of psychosocial and behavioral factors. J Gerontol B Psychol Soc Sci 2011; 66 Suppl 1:i130-40.

16. Robert SA, Cherepanov D, Palta M, Durham NC, Feeny D, Fryback DG. Socioeconomic status and age variations in health-related quality of life: Results from the national health measurement study. J Gerontol B Soc Sci 2009; 64:378-89.

17. Alvarenga MRM, Oliveira MAC, Domingues MAR, Amendola F, Faccenda O. Rede de suporte social do idoso atendido por equipes de Saúde da Família. Ciênc Saúde Coletiva 2011; 16:2603-11.
18. Espinoza SE, Jung I, Hazuda H. Lower frailty incidence among Mexican American than among European American older adults: the San Antonio Longitudinal Study of Aging. J Am Geriatr Soc 2010; 58:2142-8.

19. Borim FSA, Barros MB, Neri AL. Autoavaliação da saúde em idosos: pesquisa de base populacional no Município de Campinas, São Paulo, Brasil. Cad Saúde Pública 2012; 28:769-80.

20. Rodrigues NO, Neri AL. Vulnerabilidade social, individual e programática em idosos da comunidade: dados do estudo FIBRA, Campinas, SP, Brasil. Ciênc Saúde Coletiva 2012; 17:2129-39.

21. Costa TB, Neri AL. Medidas de atividade física e fragilidade em idosos: dados do FIBRA Campinas, São Paulo, Brasil. Cad Saúde Pública 2010; 27: 1537-50.

22. Folstein M, Folstein S, McHugh P. Mini-Mental State: a practical method for grading the cognitive status of patients for the clinician. J Psychiatr Res 1975; 12:189-98.

23. Brucki SMD, Nitrini R, Caramelli P, Bertolucci PH, Okamoto IH. Sugestões para o uso do Mini-Exame do Estado Mental no Brasil. Arq Neuropsiquiatr 2003; 61:777-81.

24. Lebrão ML, Laurenti R. Saúde, bem-estar e envelhecimento: o estudo SABE no Município de São Paulo. Rev Bras Epidemiol 2005; 8:127-41.

25. Sourdet S, Rougé-Bugat ME, Vellas B, Forette F. Frailty and aging. J Nutr Health Aging 2012; 16 : 283-4.

Recebido em 15/Jun/2012

Versão final reapresentada em 09/Out/2012

Aprovado em 03/Nov/2012 